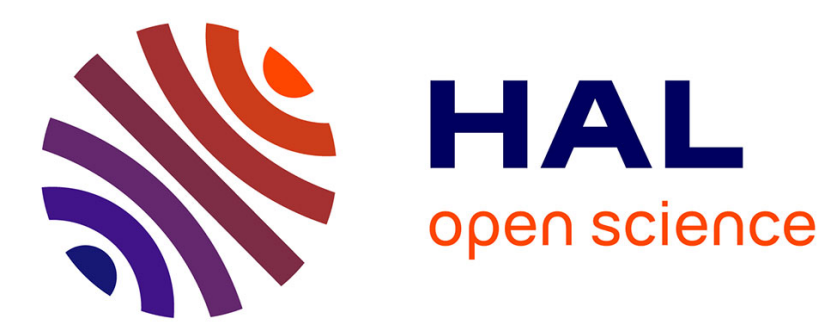

\title{
Self-induced topological transition in phononic crystals by nonlinearity management
}

Rajesh Chaunsali, Georgios Theocharis

\section{To cite this version:}

Rajesh Chaunsali, Georgios Theocharis. Self-induced topological transition in phononic crystals by nonlinearity management. Physical Review B: Condensed Matter and Materials Physics (1998-2015), 2019, 100, 10.1103/PhysRevB.100.014302 . hal-02371097

\section{HAL Id: hal-02371097 https://hal.science/hal-02371097}

Submitted on 19 Nov 2019

HAL is a multi-disciplinary open access archive for the deposit and dissemination of scientific research documents, whether they are published or not. The documents may come from teaching and research institutions in France or abroad, or from public or private research centers.
L'archive ouverte pluridisciplinaire HAL, est destinée au dépôt et à la diffusion de documents scientifiques de niveau recherche, publiés ou non, émanant des établissements d'enseignement et de recherche français ou étrangers, des laboratoires publics ou privés. 


\title{
Self-induced topological transition in phononic crystals by nonlinearity management
}

\author{
Rajesh Chaunsali* and Georgios Theocharis ${ }^{\dagger}$ \\ LAUM, CNRS, Le Mans Université, Avenue Olivier Messiaen, 72085 Le Mans, France
}

(Received 20 April 2019; published 18 July 2019)

\begin{abstract}
A design paradigm of topology has recently emerged to manipulate the flow of phonons. At its heart lies a topological transition to a nontrivial state with exotic properties. This framework has been limited to linear lattice dynamics so far. Here we show a topological transition in a nonlinear regime and its implication in emerging nonlinear solutions. We employ nonlinearity management such that the system consists of masses connected with two types of nonlinear springs, "stiffening" and "softening" types, alternating along the length. We show, analytically and numerically, that the lattice makes a topological transition simply by changing the excitation amplitude and invoking nonlinear dynamics. Consequently, we witness the emergence of a family of finite-frequency edge modes, not observed in linear phononic systems. We also report the existence of kink solitons at the topological transition point. These correspond to heteroclinic orbits that form a closed curve in the phase portrait separating the two topologically distinct regimes. These findings suggest that nonlinearity can be used as a strategic tuning knob to alter topological characteristics of phononic crystals. These also provide fresh perspectives towards understanding a different family of nonlinear solutions in light of topology.
\end{abstract}

DOI: 10.1103/PhysRevB.100.014302

\section{INTRODUCTION}

The advent of topological insulators (TIs) in condensedmatter physics has popularized a tool to characterize material dispersion [1]. This tool is the topology of energy bands. It offers a powerful framework of the bulk-boundary correspondence, in which one can characterize a bulk (infinite) material topologically, and predict the response at the boundary of its finite counterpart. At a physical level, this explains why TIs allow chiral and robust currents on their boundaries but do not support any current in their bulk. Recently, this framework has been extended beyond electronic systems and has been applied to classical systems in photonics [2] and phononics [3]. For example, acoustic and elastic topological structures show various possibilities of manipulating wave flow [4]. These have the potential to provide different solutions for applications, such as noise mitigation, vibration isolation, sensing, and energy harvesting. At the same time, these structures act as tabletop setups for investigating topological physics at a fundamental level.

The study of band topology in phononic lattices has been largely restricted to linear dynamics so far. It is not clear how this framework could be relevant for nonlinear systems as well, where wave dispersion becomes amplitude dependent, at least in weakly nonlinear regimes. Previous studies have shown the excellent potential of nonlinear phononic crystals in manipulating vibrations in general (see $[5,6]$ and references therein). Especially appealing are elastic systems in which a tremendous degree of nonlinearity management could be achieved through material and geometric parameters, for example, using contacts [7], LEGO blocks [8], origami folding [9], tensegrity structures [10], or architected soft media [11].

\footnotetext{
*rajeshcuw@gmail.com

†georgiostheocharis@gmail.com
}

All these structures and other proposed flexible mechanical metamaterials [12] can thus be excellent model candidates for the fundamental understanding of the interplay of nonlinearity and topology in mechanics. At the same time, the findings can be experimentally verified, and also supplement other active areas, such as nonlinear photonics, where exciting theoretical advances have been made recently [13-21], but with limited experimental success [19,22].

One may ask why is the study of nonlinearity relevant in topological systems after all. In this quest, the recent studies in both photonics and phononics have shown two broad pathways. The first is to assess the effect of nonlinearity, inevitable in most systems at large operating amplitudes, on the topologically robust edge states present in a linear system. For example, one can investigate the change in frequency and stability of edge modes $[16,19,22,23]$, or reveal the formation of topologically robust edge solitons $[14,15,24]$. The second pathway is to explore strategically designed nonlinear lattices, in which different nonlinear solutions emerge and those are intrinsically topological in nature such that unique gap solitons $[13,18]$, and "self-induced" edge solitons [15] and domain walls $[20,25,26]$ appear in the lattice. We focus on the latter category since it provides interesting ways to complement topological effects by using nonlinearity management and at the same time provides a way to interpret emerging nonlinear solutions in light of topology.

In phononics, one such work [25] studied the dynamics of zero-frequency floppy modes in a one-dimensional (1D) mechanical chain, the exact mapping of the celebrated SuSchrieffer-Heeger (SSH) model $[27,28]$. It was shown that these modes can travel from one end to the other in the form of a soliton along the chain, and the soliton is interpreted as a moving domain wall in a topological sense. However, when it comes to topological band theory for finite (nonzero)frequency modes [3] - relevant for vibration studies-it is not clear how emergent nonlinear solutions, such as 


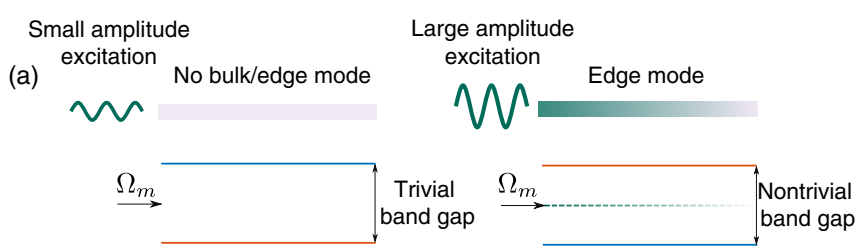

(b)

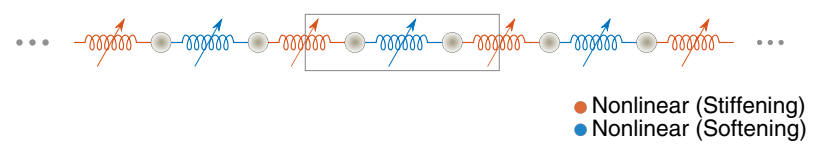

FIG. 1. (a) A schematic of the "self-induced" topological transition in the system. For small amplitude excitation, the system has a trivial band gap, with no edge or bulk mode at the midgap frequency $\Omega_{m}$. However, for large amplitude, nonlinearity kicks in and forces the system to a nontrivial state. This leads to the emergence of an edge mode. (b) A chain of point masses and nonlinear springs (stiffening in red and softening in blue) is designed to induce the aforementioned effect. The box highlights the unit cell.

"self-induced" solitons and domain wall, are connected with band topology of phonons.

To address this question, here, we consider one of the most fundamental mechanisms in topological band theorythe band inversion. We take a phononic crystal, a chain made of masses and two alternating springs, and ask a question: Can we engineer a nonlinear phononic lattice that shows a band inversion, a topological transition, and support emergent edge modes solely based on the amplitude of vibration excitation [Fig. 1(a)]? The model is inspired from the SSH chain [27], and the difference lies in the fact that spring stiffnesses contribute to the diagonal elements of the bulk Hamiltonian, and therefore, the band gap is centered at a finite frequency [29]. If this "self-induced" topological transition could be engineered, we expect to observe a family of emerging nonlinear solutions with the increase in excitation amplitude and thus establish any connection they might have with the topological transition. By introducing nonlinearity in all the springs, different from the previous studies $[17,30]$, we extensively rely on phase portrait analysis to unveil a rich nonlinear dynamics in the system. In this process, we discover stationary kink soliton solutions, and those can be interpreted as a "self-induced" domain wall separating two topologically distinct regions within the purview of an "effective" topological band theory of our nonlinear lattice. Moreover, we study how nonlinear effects lead to unique edge solutions in the system depending on excitation amplitude and how this fact could be used for designing tunable topological systems.

We organize this paper as follows: In Sec. II, we propose a scheme of nonlinearity management, using a combination of "stiffening" and "softening" type of nonlinear springs with cubic nonlinearity. In Sec. III, we describe amplitude-dependent dispersion properties and general solutions, and show the mechanism of a band inversion and thus a topological transition. In Sec. IV, we employ analytical techniques and use phase portrait to reveal emerging nonlinear solutions in the bulk, such as nonlinear evanescent modes and kink solitons. We establish a connection between these nonlinear solutions and band topology. In Sec. V, we use numerical techniques to verify their existence for finite structures. In Sec. VI, for experimental feasibility, we also extend these results for a "saturating" type of nonlinearity and show how this system can support an in situ emergence of an edge mode due to a topological transition. In Sec. VII, we conclude our findings and provide fresh perspectives towards extending this work to different topological systems and also realizing it in experiments.

\section{SYSTEM DETAILS}

Figure 1(b) shows the system configuration, which is a chain of point masses connected with nonlinear springs. We use a "stiffening" and "softening" type of springs alternatively along the chain. The unit cell consists of two masses. For the first section of this paper, we take the following forcedeformation profiles with cubic nonlinearity for two types of springs:

$$
\begin{aligned}
& F(\Delta x)=(1-\gamma) k \Delta x+k_{3}(\Delta x)^{3} \quad \text { (stiffening), } \\
& F(\Delta x)=(1+\gamma) k \Delta x-k_{3}(\Delta x)^{3} \quad \text { (softening), }
\end{aligned}
$$

where $\Delta x$ and $F$ are spring deformation and force, respectively; $k>0$ and $k_{3}>0$ are the stiffness coefficients; $\gamma>0$ denotes the mismatch in stiffness for small amplitude (linear dynamics) and this determines the width of the initial band gap. We can thus write equations of motion neglecting any dissipation as

$$
\begin{aligned}
m \frac{d^{2} x_{1, n}}{d t^{2}}= & F\left(x_{2, n-1}-x_{1, n}\right)-F\left(x_{1, n}-x_{2, n}\right) \\
= & (1-\gamma) k\left(x_{2, n-1}-x_{1, n}\right)+k_{3}\left(x_{2, n-1}-x_{1, n}\right)^{3} \\
& -(1+\gamma) k\left(x_{1, n}-x_{2, n}\right)+k_{3}\left(x_{1, n}-x_{2, n}\right)^{3}, \\
m \frac{d^{2} x_{2, n}}{d t^{2}}= & F\left(x_{1, n}-x_{2, n}\right)-F\left(x_{2, n}-x_{1, n+1}\right) \\
= & (1+\gamma) k\left(x_{1, n}-x_{2, n}\right)-k_{3}\left(x_{1, n}-x_{2, n}\right)^{3} \\
& -(1-\gamma) k\left(x_{2, n}-x_{1, n+1}\right)-k_{3}\left(x_{2, n}-x_{1, n+1}\right)^{3},
\end{aligned}
$$

where $x_{1, n}$ and $x_{2, n}$ denote displacements of two particles inside the $n$th unit cell. Let the unit-cell length be $a$ and $\Gamma=a^{2} k_{3} / k$. Therefore, we can nondimensionalize equations of motion by defining new variables $\tau=t \sqrt{k / m}$ and $\xi=x / a$, and write

$$
\begin{aligned}
\ddot{\xi}_{1, n}= & (1-\gamma)\left(\xi_{2, n-1}-\xi_{1, n}\right)+\Gamma\left(\xi_{2, n-1}-\xi_{1, n}\right)^{3} \\
& -(1+\gamma)\left(\xi_{1, n}-\xi_{2, n}\right)+\Gamma\left(\xi_{1, n}-\xi_{2, n}\right)^{3}, \\
\ddot{\xi}_{2, n}= & (1+\gamma)\left(\xi_{1, n}-\xi_{2, n}\right)-\Gamma\left(\xi_{1, n}-\xi_{2, n}\right)^{3} \\
& -(1-\gamma)\left(\xi_{2, n}-\xi_{1, n+1}\right)-\Gamma\left(\xi_{2, n}-\xi_{1, n+1}\right)^{3} .
\end{aligned}
$$

For the rest of the paper, we stick to this nondimensionalized framework, where $\Gamma, \tau, \xi$, and $\Delta \xi$ denote nonlinearity parameter, time, displacement, and strain, respectively.

\section{BAND-GAP SOLUTIONS AND TOPOLOGICAL TRANSITION WITH AMPLITUDE}

\section{A. Band-gap solutions}

In the linear limit, the system represents a periodic chain consisting of two alternating springs with stiffness $1-\gamma$ and $1+\gamma$. The dispersion relation, as shown in Fig. 2(a), consists 
(a)

(b) $\Omega^{2}$
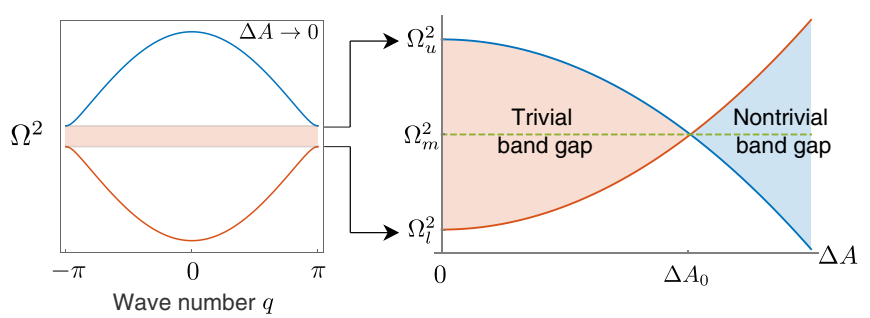

FIG. 2. (a) Dispersion relation of the unit cell with small strain amplitude $\Delta A$, a linear regime. It highlights a band gap between acoustical (lower) and optical (upper) branches. (b) Increase in strain amplitude $\Delta A$ changes the band gap, by closing it first and then opening again to a nontrivial state.

of two branches, the acoustical (lower) with $\Omega_{l}^{2}=2(1-\gamma)$ at $q= \pm \pi$, and the optical (upper) with $\Omega_{u}^{2}=2(1+\gamma)$ at $q=$ $\pm \pi$. Here $q$ represents the normalized wave number. Thus the system has a band gap of width $\Omega_{u}^{2}-\Omega_{l}^{2}$. It is straightforward to show that the eigenmode corresponding to $\Omega_{l}^{2}$ is given by $\left(\xi_{1, n}, \xi_{2, n}\right)=(A, A) \exp ($ iqn $)=(-1)^{n}(A, A)$, where $A$ represents the amplitude of oscillation. Physically, this means that the two particles inside the unit cell oscillate in phase. Similarly, the eigenmode at $\Omega_{u}^{2}$ is given by $\left(\xi_{1, n}, \xi_{2, n}\right)=$ $(A,-A) \exp ($ iqn $)=(-1)^{n}(A,-A)$ representing the out-of-phase motion of particles inside the unit cell.

In this work, we focus on the weakly nonlinear wave solutions, i.e., $\Gamma=O(\epsilon)$ inside a small band gap with $\gamma=O(\epsilon)$. Therefore we can look for slowly varying solutions around frequency $\Omega$ with the following ansatz:

$$
\begin{aligned}
& \xi_{1, n}=\frac{(-1)^{n}}{2}\left[u(n, t) \exp (i \Omega \tau)+u^{*}(n, t) \exp (-i \Omega \tau)\right], \\
& \xi_{2, n}=\frac{(-1)^{n}}{2}\left[v(n, t) \exp (i \Omega \tau)+v^{*}(n, t) \exp (-i \Omega \tau)\right],
\end{aligned}
$$

where * indicates the complex conjugation and $\Omega \in$ $\left[\Omega_{l}, \Omega_{u}\right]$. We substitute the ansatz in Eq. (2) and make continuum approximation. To this end, we choose $n=$ $z, u(n-1, t)=u(z, t)-\partial u(z, t) / \partial z, u(n+1, t)=u(z, t)+$ $\partial u(z, t) / \partial z, \quad v(n-1, t)=v(z, t)-\partial v(z, t) / \partial z$, and $v(n+$ $1, t)=v(z, t)+\partial v(z, t) / \partial z$, where the derivatives are small of $O(\epsilon)$. We ignore higher harmonics and collect the terms of $O(\epsilon)$ to obtain the following PDEs:

$$
\begin{aligned}
-4 i \Omega \frac{\partial u(z, t)}{\partial t}= & -2 \frac{\partial v(z, t)}{\partial z}+2\left(2-\Omega^{2}\right) u(z, t)-4 \gamma v(z, t) \\
& +3 \Gamma\left[2|u(z, t)|^{2}+|v(z, t)|^{2}\right] v(z, t) \\
& +3 \Gamma u(z, t)^{2} v^{*}(z, t), \\
-4 i \Omega \frac{\partial v(z, t)}{\partial t}= & 2 \frac{\partial u(z, t)}{\partial z}+2\left(2-\Omega^{2}\right) v(z, t)-4 \gamma u(z, t) \\
& +3 \Gamma\left[|u(z, t)|^{2}+2|v(z, t)|^{2}\right] u(z, t) \\
& +3 \Gamma v(z, t)^{2} u^{*}(z, t) .
\end{aligned}
$$

For steady-state solutions, we can simply substitute $u(z, t)=u(z)=u$ and $v(z, t)=v(z)=v$ in Eq. (4) and deduce the following ODEs:

$$
\begin{aligned}
& u^{\prime}=-\left(2-\Omega^{2}\right) v+2 \gamma u-\frac{3}{2} \Gamma\left(u^{3}+3 u v^{2}\right), \\
& v^{\prime}=\left(2-\Omega^{2}\right) u-2 \gamma v+\frac{3}{2} \Gamma\left(v^{3}+3 u^{2} v\right),
\end{aligned}
$$

where ' denotes first derivative with respect to $z$, and $u$ and $v$ are real quantities.

\section{B. Band-gap closure with amplitude}

We calculate the nonlinear continuation of the lower and upper band-edge modes by substituting $u=v=A$ for $\Omega=$ $\Omega_{l}$, and $u=-v=A$ for $\Omega=\Omega_{u}$ in Eq. (5). These represent steady-state and spatially periodic solutions with amplitude $A$. We use the fact that for these modes, strain amplitudes $(\triangle A)$ are $2 A$ and 0 for the two springs inside the unit cell, therefore

$$
\begin{aligned}
& \Omega_{l}^{2}(\Delta A)=2(1-\gamma)+\frac{3}{2} \Gamma(\Delta A)^{2}, \\
& \Omega_{u}^{2}(\Delta A)=2(1+\gamma)-\frac{3}{2} \Gamma(\Delta A)^{2} .
\end{aligned}
$$

We plot the band-edge continuation in Fig. 2(b). We see that the band gap first closes at $\Delta A_{0}=2 \sqrt{\gamma / 3 \Gamma}$ and then opens again, with band-edge frequencies flipped—indicating a band inversion. Note that such a band inversion could be achieved by other nonlinearity management, for example, by taking linear and nonlinear springs alternating along the chain as was done in photonics [17]. However, by using a combination of stiffening and softening type of nonlinear springs in this study, we not only achieve a band inversion, but also we make sure that the midgap solutions (point of interest in 1D topological systems) occur at the same frequency, $\Omega_{m}^{2}=\left[\Omega_{l}^{2}(\Delta A)+\Omega_{u}^{2}(\Delta A)\right] / 2=2$, for different amplitude excitations.

\section{Topological transition}

One can characterize the band topology of a onedimensional linear system using the so-called Zak phase $[31,32]$. This is the geometric phase accumulated by the Bloch wave vector when transported adiabatically along a frequency band in the first Brillouin zone. For a linear dimer system with inversion symmetry, it is either 0 or $\pi$. For the unit cell shown in Fig. 1(b) but made of linear springs, one can easily verify that it would have a nonzero Zak phase $\pi$ when the stiffness of red spring is larger than the stiffness of blue spring. In other words, a finite-frequency SSH model would have a nonzero Zak phase when intercell stiffness ( $\left.K_{\text {inter }}\right)$ would be larger than intracell stiffness $\left(K_{\text {intra }}\right)$, such that [29]

$$
\mathcal{Z}=\frac{\pi}{2}\left[1+\operatorname{sgn}\left(K_{\text {inter }}-K_{\text {intra }}\right)\right],
$$

where $\operatorname{sgn}($.$) denotes the sign function. Now the question is$ how could we utilize this formulation of the Zak phase in a nonlinear system? The question is highly nontrivial since Bloch vectors become amplitude dependent. This implies that the geometric phase accumulated by the Bloch vector along a frequency band could then vary depending on how much amplitude excitation we give at each frequency, and this fact 
(a)

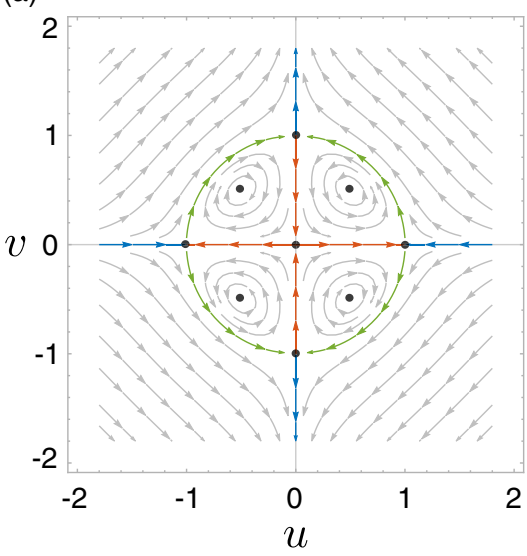

(b)

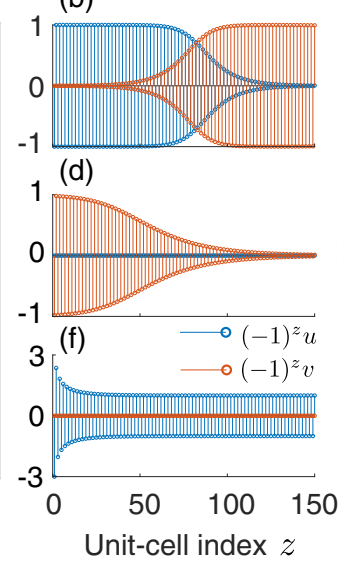

(c)

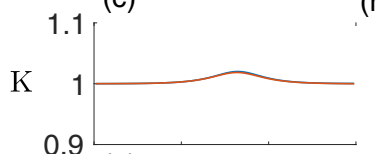

(e)

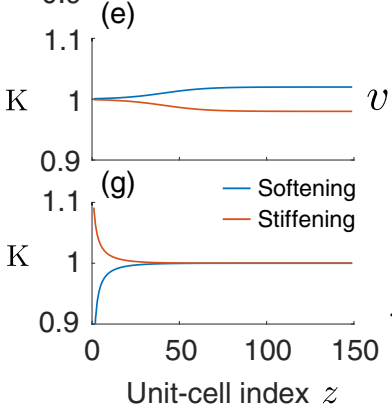

(h)

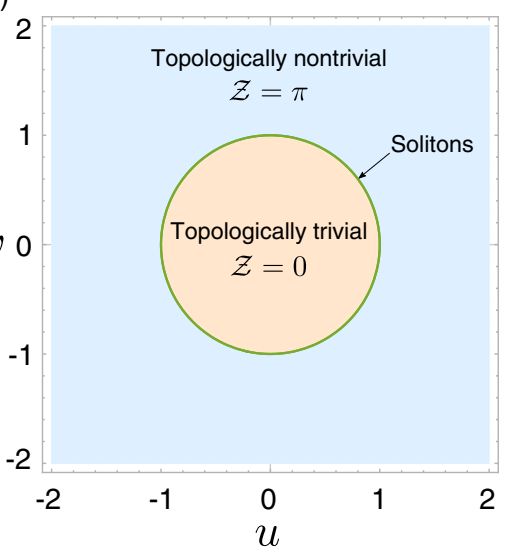

FIG. 3. (a) Phase portrait of the bulk showing steady-state solutions at the midgap frequency. It highlights nine fixed points in black dots. The arrows in red, blue, and green indicate the presence of multiple evanescent and soliton solutions. (b) Soliton profile that corresponds to the heteroclinic orbits in green in the phase portrait. (c) Effective stiffness for all the springs along the chain, calculated for the aforementioned mode profile. This indicates the closure of "local band gap." (d),(e) For small amplitude regimes, a profile and its effective stiffness, respectively, extracted from one of the evanescent solutions shown in red in the phase portrait. Note the amplitude forms a plateau towards the left edge. (f),(g) For large amplitude regimes, a profile and its effective stiffness, respectively, extracted from one of the evanescent solutions shown in blue in the phase portrait. Note the amplitude forms a plateau towards the right edge. (h) A schematic to show topologically distinct regions in the phase portrait that are separated by the circular trajectory of solitons.

would defeat the basic definition of a bulk invariant. Therefore, we argue that such a nonlinear system would not have a bulk invariant in the sense of a linear system. However, for a weakly nonlinear system such as ours, where the Bloch theorem is still valid, we map our nonlinear system to an effective linear system at the unit-cell level. For nonlinear excitations inside the small band gap, we thus write effective stiffnesses for the two types of nonlinear springs (see Appendix A for more discussion):

$$
\begin{aligned}
& K_{\text {stiffening }}(\Delta A)=\frac{\Omega_{l}^{2}(\Delta A)}{2}=(1-\gamma)+\frac{3}{4} \Gamma(\Delta A)^{2}, \\
& K_{\text {softening }}(\Delta A)=\frac{\Omega_{u}^{2}(\Delta A)}{2}=(1+\gamma)-\frac{3}{4} \Gamma(\Delta A)^{2} .
\end{aligned}
$$

We thus characterize this mapped linear system by

$$
\mathcal{Z}(\Delta A)=\frac{\pi}{2}\left\{1+\operatorname{sgn}\left[K_{\text {stiffening }}(\Delta A)-K_{\text {softening }}(\Delta A)\right]\right\} .
$$

By using the expressions in Eq. (7), our mapped system, therefore, makes a transition from a topologically trivial state $(\mathcal{Z}=0)$ to a topologically nontrivial state $(\mathcal{Z}=\pi)$ when strain amplitude crosses $\Delta A_{0}$ and bands are inverted. One may, however, notice another assumption in this approach, and that is to assume the equal strain amplitude $\Delta A$ in all the springs. To explain the existence of any edge or soliton solution using this approach is not straightforward since such nonuniform spatial solutions in a nonlinear system as ours can change effective stiffness, locally, along the chain and the system no longer remains periodic from the effective stiffness point of view. Unit-cell description, fundamental in characterizing global topology, thus breaks down. Then the question is whether this simplified description of topology is of any use in the current case? The answer is yes. We will show in the sections below that we do observe soliton and edge solutions in the system, whose presence could be explained by an amplitude-dependent topological transition if topological characterization is done locally by calculating local effective stiffness.

\section{PHASE PORTRAIT AND EMERGENCE OF SOLITON AND EVANESCENT SOLUTIONS}

\section{A. Midgap steady-state solutions}

From now onward, we focus on the solutions at the midgap frequency $\Omega_{m}^{2}=2$. Therefore, Eq. (5) reduces to

$$
\begin{aligned}
& u^{\prime}=2 \gamma u-\frac{3}{2} \Gamma\left(u^{3}+3 u v^{2}\right), \\
& v^{\prime}=-2 \gamma v+\frac{3}{2} \Gamma\left(v^{3}+3 u^{2} v\right) .
\end{aligned}
$$

These describe the dynamics of a Hamiltonian system with a conserved energy

$$
E=2 \gamma u v\left[1-\frac{3 \Gamma}{4 \gamma}\left(u^{2}+v^{2}\right)\right],
$$

where $u$ and $v$ may be considered as generalized coordinate and momentum, respectively. Using these equations, we now plot the phase portrait in Fig. 3(a) with $4 \gamma=3 \Gamma=0.08$ and look for special trajectories.

We observe nine fixed points $\left(u^{\prime}=v^{\prime}=0\right)$ highlighted by black dots at $(u, v)=(0,0),( \pm 2 \sqrt{\gamma / 3 \Gamma}, 0),(0, \pm 2 \sqrt{\gamma / 3 \Gamma})$, and $( \pm \sqrt{\gamma / 3 \Gamma}, \pm \sqrt{\gamma / 3 \Gamma})$. Four of them, the ones at $( \pm 2 \sqrt{\gamma / 3 \Gamma}, 0),(0, \pm 2 \sqrt{\gamma / 3 \Gamma})$, are connected by heteroclinic orbits forming a circular curve in the phase portrait. Notice that the strain amplitude at these fixed points is exactly the same as $\Delta A_{0}$ shown in Fig. 2(b). As we will see next, this circle separates the phase portrait into two topologically distinct regions. For the choice of the unit cell, highlighted in Fig. 1(b) by the box, the region inside (outside) the circle 
corresponds to a topologically trivial (nontrivial) band gap. Below we study in detail solutions corresponding to the two topologically distinct regimes as well as solutions at the topological transition on the circle.

\section{B. Topological transition point: Bulk soliton solutions}

In Fig. 3(b), we plot the solution corresponding to the circular trajectory (in green) in the first quadrant of the phase portrait. These are soliton solutions and represent two kinks of opposite polarities. On the left end of the chain, the unit cell has only the first mass moving (predominately $u$ component) while towards the right its polarity changes and only the second mass vibrates (predominantly $v$ component). Interestingly enough, a similar soliton solution has been observed experimentally [33] and studied analytically in some earlier works $[34,35]$, but in different types of nonlinear lattices. We calculate the analytical expression of the soliton profile by finding that the soliton trajectories correspond to $E=0$ in Eq. (10) and thus would have the locus given by

$$
u^{2}+v^{2}=\frac{4 \gamma}{3 \Gamma}
$$

We can further deduce (details in Appendix B)

$$
\begin{aligned}
& u= \pm \sqrt{\frac{2 \gamma}{3 \Gamma}[1 \pm \tanh (4 \gamma z)]}, \\
& v= \pm \sqrt{\frac{2 \gamma}{3 \Gamma}[1 \mp \tanh (4 \gamma z)]} .
\end{aligned}
$$

These solutions represent four soliton trajectories in all four quadrants of the phase portrait.

How could we explain these soliton solutions on the ground of topology? To this end, we calculate effective stiffness for each spring along the chain for these soliton solutions. We find them remarkably close to each other, as shown in Fig. 3(c). This indicates that the "local band gap," dictated by two consecutive effective stiffnesses, closes along the entire chain. This, therefore, hints at a local topological transition as per Eq. (8). We can prove it analytically in the following way. For displacements $u$ and $v$ inside a unit cell, its neighboring unit cells would have the displacements $-\left(u \pm u^{\prime}\right)$ and $-\left(v \pm v^{\prime}\right)$. Strain amplitudes in two consecutive springs would thus be $\Delta A$ (softening) $=|u-v|$ and $\Delta A$ (stiffening) $=\left|u+v+u^{\prime}\right|$ or $\left|u+v-v^{\prime}\right|$. For the local band gap to close, we need $K_{\text {stiffening }}=K_{\text {softening }}$ for this unit cell. Therefore, from Eq. (7), we deduce

$$
2 \gamma-\frac{3}{4} \Gamma(u-v)^{2}-\frac{3}{4} \Gamma\left(u+v+u^{\prime}\right)^{2}=0 .
$$

Since $\gamma, \Gamma$, and $u^{\prime}$ are small quantities, we neglect their higher power and deduce the condition for the closure of local band gap,

$$
u^{2}+v^{2}=\frac{4 \gamma}{3 \Gamma}
$$

This condition is exactly the same as the soliton locus in the phase portrait given by Eq. (11) and proves the claim that soliton profiles allow effective stiffness distribution in such a way that the local band gap closes along the entire chain. On a more intuitive level, we see the soliton in Fig. 3(b) as a solution consisting of two plane waves towards the left and right extremities, and those individually represent the closure of the band gap. We conjecture that the soliton solution can be thought of as a symmetry-breaking solution that transitions between these two plane waves of opposite polarities and maintains the band-gap closure, locally. It is straightforward to conclude that we would have $K_{\text {stiffening }}<K_{\text {softening }}$, locally, along the chain for $u^{2}+v^{2}<4 \gamma / 3 \Gamma$. This is the region inside the circle and corresponds to small amplitudes. The region is topologically trivial with $\mathcal{Z}=0$. In the same way, the region outside the circle, representing large amplitudes, could be shown to have $K_{\text {stiffening }}>K_{\text {softening }}$, locally, along the chain. This would be topologically nontrivial with $\mathcal{Z}=\pi$.

\section{Topologically trivial solutions}

For the topologically trivial band gap, we focus on evanescent solutions [in red in Fig. 3(a)]. These connect the origin $(u, v)=(0,0)$ to the neighboring four fixed points. Their profiles would decay to zero amplitude in one direction, similar to the linear evanescent mode shown in Appendix C, but saturate to a constant in the opposite direction. This constant is dictated by a fixed point in the phase portrait. It is easy to verify that the strain amplitude corresponding to this fixed point is the same $\Delta A_{0}$ we see in Fig. 2(b). We extract one such solution with the initial condition at $\left(u_{0}, v_{0}\right)=(0,0.98)$ and plot displacements in Fig. 3(d). We can also derive an analytical form of this solution by substituting $u=0$ in Eq. (9) and solving for the initial condition $(u, v)=\left(0, v_{0}\right)$ at $z=z_{0}$ (details in Appendix B), and we get

$$
\begin{aligned}
& u=0 \\
& v= \pm\left[\frac{3 \Gamma}{4 \gamma}+\left(\frac{1}{v_{0}^{2}}-\frac{3 \Gamma}{4 \gamma}\right) \exp \left[4 \gamma\left(z-z_{0}\right)\right]\right]^{-1 / 2} .
\end{aligned}
$$

Clearly, for the initial condition $\left|v_{0}\right|<2 \sqrt{\gamma / 3 \Gamma}$, we have $|v|$ decreasing to zero as $z \rightarrow \infty$. In the linear limit $(\Gamma \rightarrow 0)$, this mode profile converges to the conventional evanescent solutions observed in linear systems. We can easily calculate strain amplitude in each spring for this mode and verify $K_{\text {stiffening }}<K_{\text {softening }}$ along the entire chain [Fig. 3(e)] using Eq. (7), confirming a topologically trivial solution. See also the comment on four other fixed points and neighboring periodic orbits within this region of the phase portrait in Appendix D.

\section{Topologically nontrivial solutions}

We now discuss the large amplitude solutions lying outside the circle in the phase portrait. For example, the trajectory in blue that lies on the $v=0$ axis emerges from $u= \pm \infty$ and asymptotically converges to the fixed points $(u, v)=( \pm 2 \sqrt{\gamma / 3 \Gamma}, 0)$, whereas the trajectory in blue that lies on the $u=0$ axis emerges from the fixed points $(u, v)=$ $(0, \pm 2 \sqrt{\gamma / 3 \Gamma})$ and asymptotically converges to $v= \pm \infty$. To visualize one such solution, we take $\left(u_{0}, v_{0}\right)=(3,0)$ as an initial condition and plot displacements in Fig. 3(f). Different from the evanescent solutions in linear systems that exponentially decay to zero in the bulk (see Appendix C), here we see another unique nonlinear solution that decays to form a plateau in the bulk. Strain amplitude at this plateau is the same 
as $\Delta A_{0}$ in Fig. 2(b). Such a mode is observed in a photonic lattice with alternating linear and nonlinear interactions in the SSH chain [17]. We can also derive an analytical form of this solution by substituting $v=0$ in Eq. (9) and solving for the initial condition $(u, v)=\left(u_{0}, 0\right)$ at $z=z_{0}$ (details in Appendix B), and it is given by

$$
\begin{aligned}
& u= \pm\left[\frac{3 \Gamma}{4 \gamma}+\left(\frac{1}{u_{0}^{2}}-\frac{3 \Gamma}{4 \gamma}\right) \exp \left[-4 \gamma\left(z-z_{0}\right)\right]\right]^{-1 / 2} \\
& v=0
\end{aligned}
$$

where the negative sign refers to another solution in the phase portrait with negative $u_{0}$ as the initial condition. We can verify that for the initial condition $\left|u_{0}\right|>2 \sqrt{\gamma / 3 \Gamma},|u|$ decreases when $z$ increases, and it saturates to $2 \sqrt{\gamma / 3 \Gamma}$ for $z \rightarrow \infty$. There is no linear limit for such a nonlinear mode since for $\Gamma \rightarrow 0$ the mode is pushed to infinite amplitude with a plateau at infinite. After calculating strain amplitude in each spring, we use Eq. (7) to plot effective stiffness along the chain in Fig. 3(g). As discussed above, we verify that $K_{\text {stiffening }}>K_{\text {softening }}$ all along the chain, confirming a topologically nontrivial solution.

Therefore, we can derive an interpretation of topology directly from the phase portrait and show it as a schematic in Fig. 3(h). For small amplitudes, near the origin, the bulk has a topologically trivial band gap. It hosts a unique nonlinear evanescent mode shown in Fig. 3(d). Whereas for large amplitudes, the bulk has a topologically nontrivial band gap containing a different looking evanescent mode as shown in Fig. 3(f). Finally, for intermediate amplitudes, a band inversion occurs such that there exist soliton solutions that locally close the band gap along the chain, thereby forming a "self-induced" domain wall between two topologically distinct regions. Note that the topological classification is dependent on the choice of the unit cell here. By flipping the springs in the unit cell, the region inside (outside) the soliton trajectory in the phase portrait would change to a topologically nontrivial (trivial) region. The essential point is to recognize the topological distinction between the outer and the inner regions. In the following section, we would use full numerical simulations to demonstrate the existence of the bulk nonlinear solutions seen in this section for finite chain settings.

\section{NUMERICAL VERIFICATION}

We enforce a fixed-fixed boundary condition and take 299 masses along the chain. We treat the system as semi-infinite, and therefore we only focus on the edge solution on the left boundary. We first consider a case of a symmetry-preserving boundary, in which the boundary does not cut our chosen unit cell. We, therefore, expect that the bulk-boundary correspondence would apply and only the topologically nontrivial bulk would support an edge mode $[32,36]$. We use the solutions shown in Figs. 3(b), 3(d), and 3(f) as initial conditions for the chain and numerically solve Eq. (2).

Figures 4(a)-4(c) show the resulting spatiotemporal diagram for the absolute value of strain in each spring. We choose this quantity for better visualization of 3D plots with minimal oscillatory components. The mode profile [Fig. 3(d)], representing a small amplitude regime, shows significant scattering

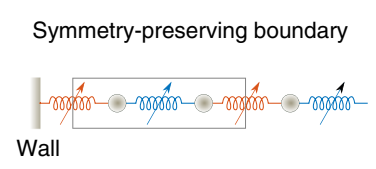

(a)
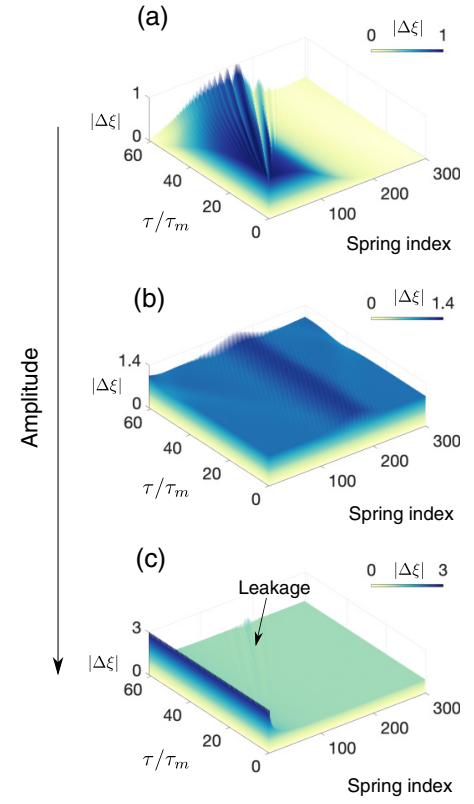
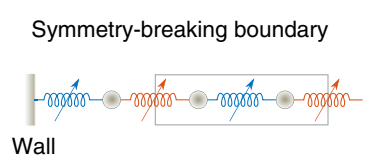

(d)
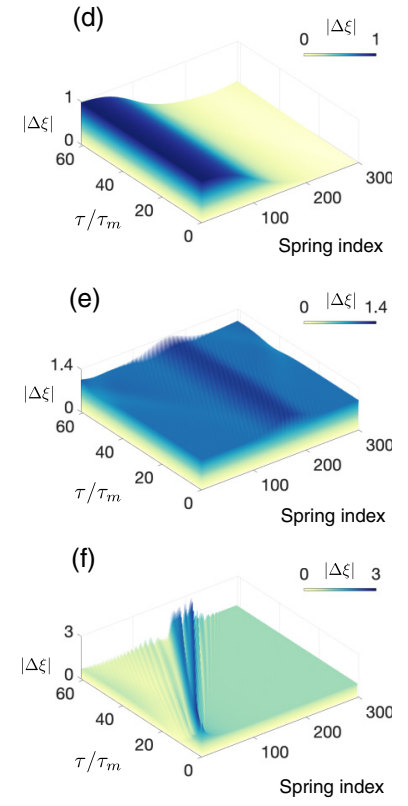

FIG. 4. Full numerical simulations verifying which bulk solutions exist in a finite chain with specific boundary condition. (a)-(c) Solutions for the boundary condition that preserves the lattice symmetry. These show space-time evolution of the absolute value of strain $|\Delta \xi|$ with the initial conditions obtained from the bulk solutions in Figs. 3(d), 3(b), and 3(f), respectively. (d)-(f) The same but for the boundary condition that breaks the lattice symmetry. $\tau_{m}=2 \pi / \Omega_{m}$ represents the time period of oscillations at the midgap frequency. Spring index 1 denotes the spring attached to the wall. Note that we are not interested in seeing the effect of boundary on the soliton solutions in (b) and (e), hence those are kept the same.

in its time evolution in Fig. 4(a). As predicted before, this evanescent solution belongs to a topologically trivial bulk, and therefore it does not lead to a nonlinear mode for this finite chain. We then show the time evolution of the soliton profile [Fig. 3(b)] in Fig. 4(b). This represents an intermediate amplitude regime. We do not observe any significant radiation in this case, confirming that this is a solution of the finite chain. We assign the cause of small radiation to the discreteness and a possible instability. Finally, for large amplitude regimes, we plot the space-time evolution of the mode profile [Fig. 3(f)] in Fig. 4(c). It is reasonably stable initially, and thus, it confirms the existence of this mode for the finite chain. This demonstrates the bulk-boundary correspondence as the bulk is topologically nontrivial in this regime. We do observe some leakage emanating from the sharp localized peak on the boundary. We assign this error to the fact that the mode profile is obtained using continuum approximation, which assumes a slow variation of displacement amplitude along the chain. Therefore, it is expected to be less accurate for the sharp localized peak on the boundary.

Now we consider a finite chain with a symmetry-breaking boundary condition, which cuts our chosen unit cell in half. 
This case is equivalent to having a symmetry-preserving boundary condition, but with a "new" unit cell that has two types of springs flipped. Therefore, we expect that only the topologically trivial solution shown in Fig. 3(d) would lead to a nonlinear edge solution. In Figs. 4(d)-4(f), we plot the time evolution of the three mode profiles for such a boundary. Clearly, we observe just the opposite trend. We see that the evanescent mode, belonging to a small amplitude regime, does lead to a nonlinear mode of the finite chain as shown in Fig. 4(d). However, the evanescent mode, belonging to a large amplitude regime, shows significant scattering, and thus, it does not lead to a nonlinear mode as shown in Fig. 4(f). The evolution of the soliton profile in Fig. 4(e) should remain the same as it is away from the boundaries and we do not change the boundary conditions in that case.

In this way, we have demonstrated that one can utilize amplitude dependency in the system to realize nonlinear edge solutions for finite chains. Taking this idea forward, could we also demonstrate such a transition in situ, for possible experimental settings, where only boundaries are usually excited? Focusing only on symmetry-preserving boundary conditions hereafter, could we transition to a topologically nontrivial solution and excite the nonlinear edge mode of large amplitudes? For that to happen, a significant amount of transient excitation would be needed so that one can induce the given mode profile. This is where the system with a cubic type of nonlinear profile [cf. Eq. (1)] might see some limitations because the high instantaneous strain in spring could make its effective stiffness close to zero, and thus lead to stability issues. Therefore, building on the findings so far, in the latter part of this paper, we propose another nonlinear force-deformation law for springs that circumvents this issue and we demonstrate a "self-induced" edge mode by using a boundary excitation.

\section{SATURABLE NONLINEARITY: A GOOD ALTERNATIVE FOR EXPERIMENTS}

\section{A. Effective stiffness}

We take the same spring-mass system shown in Fig. 1(b), but replace spring force-deformation law as

$$
\begin{aligned}
& F(\Delta x)=(1+\gamma) k \Delta x-2 \gamma k \frac{\operatorname{erf}(v \Delta x)}{c v} \text { (stiffening), } \\
& F(\Delta x)=(1-\gamma) k \Delta x+2 \gamma k \frac{\operatorname{erf}(v \Delta x)}{c v} \text { (softening), }
\end{aligned}
$$

where "erf" denotes the error function, $v$ is a parameter to tune its profile, and $c=2 / \sqrt{\pi}$. One can verify that the stiffening spring follows $F(\Delta x) \approx(1-\gamma) k \Delta x$ for small $\Delta x$ since $\operatorname{erf}(v \Delta x) \approx c v \Delta x$, whereas for large $\Delta x, F(\Delta x) \approx$ $(1+\gamma) k \Delta x-2 \gamma k / c v$. This indicates an increase in quasistatic stiffness, i.e., stiffening, which eventually saturates to a constant. We can make a similar argument for a softening type of nonlinearity as well.

We now expand the error function as a power series and perform nondimensionalization on the equations of motion. We thus obtain the following effective stiffness for nonlinear springs as a function of strain amplitude $\Delta A$ (details in Appendix E):

$$
\begin{aligned}
& K_{\text {stiffening }}(\Delta A)=(1-\gamma)+\sum_{n=3,5, \ldots}(-1)^{(n+1) / 2} n C_{(n-1) / 2} \Gamma_{n}\left(\frac{\Delta A}{2}\right)^{n-1}, \\
& K_{\text {softening }}(\Delta A)=(1+\gamma)-\sum_{n=3,5, \ldots}(-1)^{(n+1) / 2} n C_{(n-1) / 2} \Gamma_{n}\left(\frac{\Delta A}{2}\right)^{n-1},
\end{aligned}
$$

where $C$ represents the Catalan number and $\Gamma_{n}$ is the nonlinearity parameter for the $n$ th-order nonlinearity. For a better comparison, we choose $v$ in the force profile such that the first nonlinearity parameter $\Gamma_{3}$ equals $\Gamma$ of the cubic case in the first half of the paper.

In Fig. 5(a), we plot effective stiffness calculated from Eq. (18) and compare it with the cubic case in Eq. (7). We take first 60 terms in the infinite series for the numerical calculation. For small $\Delta A$, both types of profiles are close to each other since $\Gamma_{3}=\Gamma$. But, as the amplitude increases, higher-order nonlinear terms in the saturating profile become dominant, reflecting the saturation in effective stiffness. We also observe that the saturating profiles also cross each other at a critical amplitude $\Delta A_{0} \approx 1.5$, which is larger than the one for cubic profiles $\left(\Delta A_{0}=1\right)$. This, therefore, would reflect as a different plateau level for the edge mode.

\section{B. Edge solutions}

To get the evanescent mode profile in this case, we would again use continuum approximation. Due to its complexity, we do not calculate the entire phase portrait. Rather, we extract the dynamical equations only for $u=0$ or $v=0$, on which the evanescent modes lie [see Fig. 3(a)]. For example, for $v=0$ we obtain

$$
\begin{aligned}
& u^{\prime}=2 \gamma u+2 u\left(-\Lambda_{3} u^{2}+\Lambda_{5} u^{4}-\Lambda_{7} u^{6}+\cdots\right), \\
& v^{\prime}=0
\end{aligned}
$$

where $\Lambda_{n}=n C_{\frac{n-1}{2}} \Gamma_{n} / 2^{n-1}$ with $n=\{3,5,7, \ldots\}$. It is easy to verify that when higher-order nonlinearity is ignored $\left(\Gamma_{n}=0\right.$ for $n \geqslant 5$ ), the above equation reduces to Eq. (9) with $\Gamma_{3}=\Gamma$ and $v=0$.

In Fig. 5(b), we plot the evanescent mode profile obtained from these equations with the initial condition $\left(u_{0}, v_{0}\right)=$ $(3,0)$. We clearly see a decaying mode from the left that eventually saturates to a plateau, similar to the mode profile earlier in Fig. 3(f). Again, this is the implication of the nontrivial topology of the bulk, in which the plateau level is explained by the existence of a fixed point, which corresponds to strain amplitude $\Delta A_{0} \approx 1.5$ in this case. In Fig. 5(c), we 


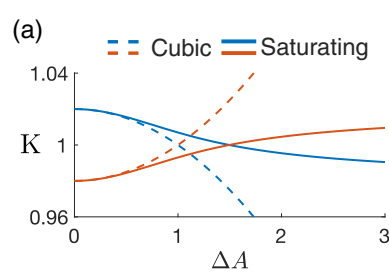

(c)

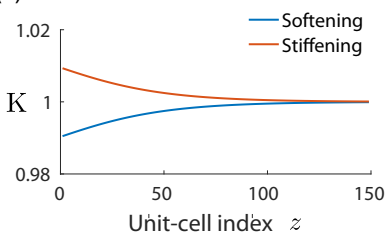

(b)

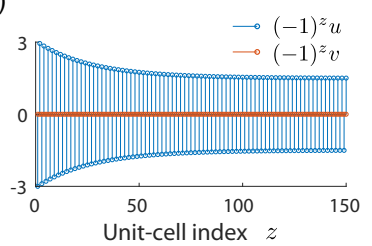

(d)

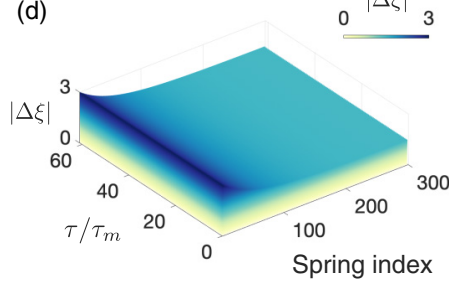

FIG. 5. (a) Effective stiffness of nonlinear springs as a function of strain amplitude $\Delta A$ when we change nonlinearity type from cubic to saturating. (b) Resulting profile of the edge mode. (c) Effective stiffness of springs along the chain for this mode. (d) Full numerical simulation showing space-time evolution of the absolute value of strain $|\Delta \xi|$ when we take the edge mode profile as the initial condition in a finite chain with the symmetry-preserving boundary.

plot the effective stiffness in each spring for the aforementioned mode profile, and it is clear that $K_{\text {stiffening }}>K_{\text {softening }}$, locally, for the entire chain. By performing full numerical simulations and plotting in Fig. 5(d), we also confirm that this evanescent mode is a solution of a finite chain with the symmetry-preserving boundary condition.

\section{In situ emergence of an edge mode}

In this section, we numerically demonstrate how an in situ topological phase transition could be achieved by increasing the excitation amplitude given at one side of the chain. As a result, we will show the emergence of an edge mode for high amplitude excitation. We apply a Gaussian-modulated sine signal in displacement, centered at the midgap frequency, to the left boundary wall of the chain. This is given as $u_{w}(\tau)=A_{w} \sin \left(\Omega_{m} \tau\right) \exp \left[-\frac{1}{2}\left(\frac{\tau-200}{70}\right)^{2}\right]$. In Fig. 6, we plot space-time evolution of strain and its frequency spectrum for three different $A_{w}$ values. For small excitation amplitude $A_{w}=0.01$, we see in Fig. 6(a) that the propagating wave is dispersive. By looking at the corresponding frequency spectrum in Fig. 6(b) calculated from time history of strain, we observe the existence of a band gap. The width of band gap also matches the blue dotted lines, which are analytically obtained cutoff frequencies in a linear dimer chain with spring coefficients $1-\gamma$ and $1+\gamma$. We do not observe any energy localization on the left end for the frequencies inside the band gap, except for the usual evanescent components, indicating a topologically trivial regime. When we increase amplitude $A_{w}$ to 1.4 , we see a distinctive wave propagation in Fig. 6(c). The wave propagation is comparatively less dispersive and is in the form of a bundle. This could correspond to a traveling soliton solution, in the form of a moving domain wall, which can be analyzed from Eq. (4). We will leave this topic for future investigations as we focus only on the stationary solutions in this study. We can see that its spectrum in Fig. 6(d) does not show any clear band gap, indicating a transition regime

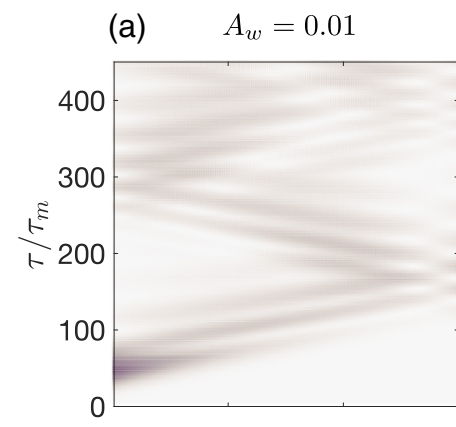

(b)

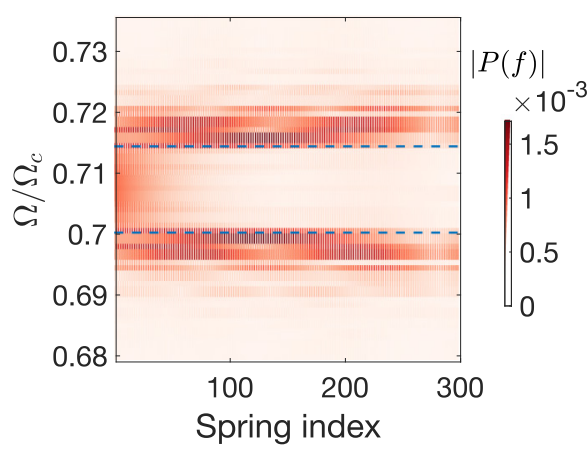

(c) $\quad A_{w}=1.4$

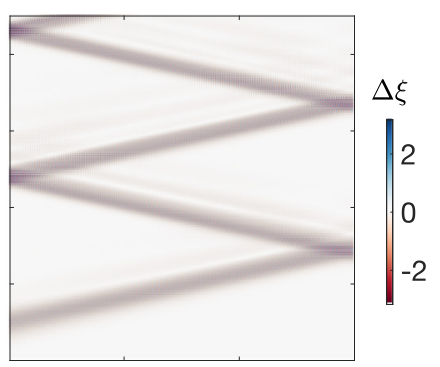

(d)

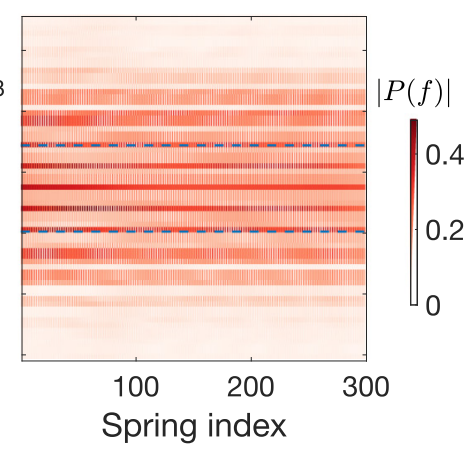

(e) $\quad A_{w}=3$

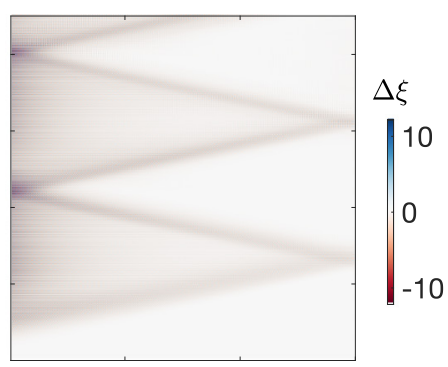

(f)

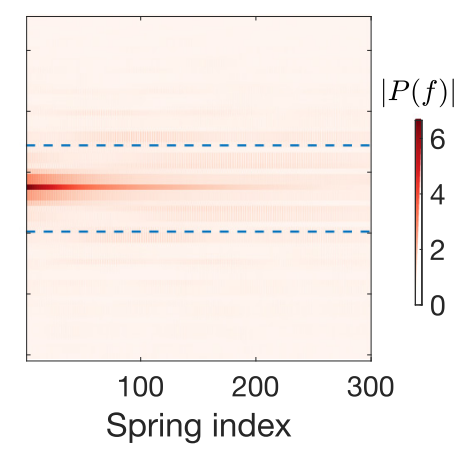

FIG. 6. (a) Space-time evolution of strain $\Delta \xi$ when we excite the left wall with a Gaussian-modulated profile of displacement amplitude $A_{w}=0.01$. (b) Spectrum calculated from the time history of spring strains for the case above. (c),(d) The same with displacement amplitude $A_{w}=1.4$. (e),(f) The same with displacement amplitude $A_{w}=3$. Spectrum plots in (b), (d), and (f) indicate the presence of a band gap, its closure, and the emergence of an edge mode, respectively. Blue dashed lines mark the lower and upper band edges for the initial (linear) configuration. $\Omega_{c}$ denotes the upper cutoff frequency of the optical branch. 
(a)

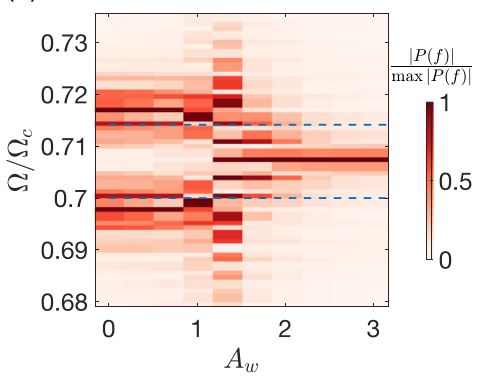

(b)

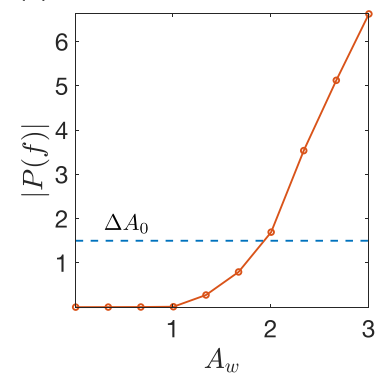

FIG. 7. (a) Normalized modal amplitude of the first spring attached to the wall as a function of excitation amplitude $A_{w}$. This shows that the emergence of an edge mode is a result of band-gap closure. (b) Modal amplitude of the first spring as a function of excitation amplitude at the midgap frequency. The critical strain amplitude $\Delta A_{0}$ is marked to indicate the point of band closure and steep increase in modal amplitude thereafter.

for band topology. Finally, for high amplitude excitation $A_{w}=3$, we remarkably observe dominant localized vibrations in Fig. 6(e) on the left side of the chain. This manifests as a clear and sharp frequency peak in the spectrum plot in Fig. 6(f).

To see this transition in the spectrum even more clearly, we plot a strain spectrum only for the first spring against a range of wall displacement amplitude in Fig. 7(a). Increasing $A_{w}$ also increases the modal amplitude $|P(f)|$, therefore, for better visualization, we choose to plot normalized modal amplitude with respect to the peak amplitude at any frequency for a given wall displacement. In this way, we can reasonably predict and observe the presence of a band gap, its closure, and the emergence of midgap-frequency spectral peak, upon increasing the excitation amplitude. This, therefore, demonstrates excitation-dependent in situ topological transition in the system. In Fig. 7(b), we plot the modal amplitude at the first spring, but only at the midgap frequency. Though a rise in modal amplitude starts around $A_{w} \approx 1$, we notice that it becomes steeper only after $A_{w} \approx 2$. This regime is remarkably close to the topological transition strain amplitude $|P(f)|=\Delta A_{0} \approx 1.5$ predicted earlier, which again confirms the fundamental topological mechanism at work.

\section{CONCLUSIONS AND FUTURE DIRECTIONS}

To summarize the main findings of this study, we successfully provide a scheme to engineer a nonlinear phononic crystal that can be transitioned to topologically distinct regimes simply by changing its dynamic excitation. The higher excitation invokes nonlinear effects in the system, and we utilize this effect to close the band gap of an initially linear system and open it again with amplitude, leading to a band inversion. We rely on a framework based on effective stiffness to show that the system makes a topological transition. Using this framework, if topological characterization is done locally, it provides a powerful tool to explain the emerging nonlinear solutions.

In the first part of this study, we consider cubic nonlinearity in alternating "stiffening" and "softening" types of springs and track steady-state solutions at the midgap frequency using a phase portrait. We witness circular heteroclinic trajectories that correspond to kink solitons. Interestingly, for these solutions the "local band gap" closes in the system and thus these represent topological transition points. In the phase portrait, this orbit separates two topologically distinct regimes in small and large amplitude regimes and can be treated as a "self-induced" domain wall. We can, therefore, utilize this amplitude dependency to transition from one topological region to another and vice versa. Depending on the choice of the unit cell and boundary, one of these regimes would be topologically nontrivial and would host a unique nonlinear edge mode, not witnessed in linear systems. Note that these observations are different from the ones reported recently in circuits [30], where a line of fixed points separated two topologically distinct regimes. This difference, in addition to the existence of more fixed points, in our case is due to the fact that we have nonlinearity in all the springs, and that gives an extra degree of freedom for the effective stiffness along the chain to vary with amplitude.

In the latter part, we build on the previous findings to suggest a system with a saturating type of nonlinearity that could potentially be a better candidate for experiments. We show that simply by exciting one end of the chain, the system transitions to a topologically nontrivial regime and thus emerges a nonlinear edge mode for large amplitudes. In principle, this nonlinearity management could be realized by utilizing geometric nonlinearity in tunable systems or performing a rigorous topology optimization of a mechanical structure.

It would be interesting to examine the stability of such nonlinear solutions in future studies. Especially, the highamplitude evanescent modes and kink solitons might radiate to low-amplitude evanescent modes in the bulk, leading to semistable behavior with a finite basin of attraction in the phase portrait [15]. If there exists a certain stability in modes, how does it relates to the robustness which is often associated with topological systems? How does discreteness affect these solutions? Could we have traveling soliton solutions in the system as Fig. 6(c) seems to suggest? It would also be interesting to extend this framework to higher dimensions, where we might expect a completely new family of solutions not witnessed in linear systems.

\section{ACKNOWLEDGMENTS}

We thank Professor D. Frantzeskakis for fruitful discussions. This work has been supported by the project CS.MICRO funded under the program Etoiles Montantes of the Region Pays de la Loire.

\section{APPENDIX A: BAND-EDGE FREQUENCIES AND EFFECTIVE STIFFNESS}

We get the nonlinear continuation of lower band-edge frequency by substituting $u=v=A$ ( $A$ being the amplitude) in Eq. (5),

$$
\Omega_{l}^{2}=2(1-\gamma)+6 \Gamma A^{2} .
$$

Clearly, this band-edge frequency increases with amplitude. This makes sense because for this mode, a softening type 
nonlinear spring does not see any strain, rather only a stiffening type spring sees a strain of amplitude $\Delta A=2 A$ [verify this by looking at the unit cell in Fig. 1(b)]. The frequency of the oscillations can thus be written in terms of effective stiffness and effective mass as $\Omega_{l}^{2}=K_{\text {stiffening }} / m_{\text {eff }}$, where $m_{\mathrm{eff}}=1 / 2$ for the unit mass chain. We can thus derive the effective stiffness for this stiffening spring in terms of strain as

$$
K_{\text {stiffening }}(\Delta A)=\frac{\Omega_{l}^{2}}{2}=1-\gamma+\frac{3}{4} \Gamma(\Delta A)^{2} .
$$

Similarly, we substitute $u=-v=A$ in Eq. (5) to get the upper band-edge frequency,

$$
\Omega_{u}^{2}=2(1+\gamma)-6 \Gamma A^{2} .
$$

We see that this band-edge frequency decreases with amplitude. This also makes sense because for this mode, a stiffening type nonlinear spring does not see any strain, rather only softening type spring sees a strain of amplitude $\Delta A=2 A$ [this can also be verified by looking at the unit cell in Fig. 1(b)]. We can thus define effective stiffness for this softening spring in terms of strain as

$$
K_{\text {softening }}(\Delta A)=\frac{\Omega_{u}^{2}}{2}=1+\gamma-\frac{3}{4} \Gamma(\Delta A)^{2} .
$$

Now one might ask if this effective stiffness framework is valid for all the solutions inside the band gap? The answer is yes, especially here when we have a small band gap and we are still looking for Bloch solutions. One of the ways to check this is to consider a linear dimer system with two springs with stiffness $K_{\text {softening }}$ and $K_{\text {stiffneing }}$ for the unit cell shown in Fig. 1(b). Let $K_{\text {softening }}>K_{\text {softening. We then follow the same }}$ approach discussed in Sec. III to derive dynamical equations for excitation frequency $\Omega$ as

$$
\begin{aligned}
K_{\text {stiffening }} u^{\prime}= & -\left(K_{\text {softening }}+K_{\text {stiffening }}-\Omega^{2}\right) v \\
& +\left(K_{\text {softening }}-K_{\text {stiffening }}\right) u \\
K_{\text {stiffening }} v^{\prime}= & \left(K_{\text {softening }}+K_{\text {stiffening }}-\Omega^{2}\right) u \\
& -\left(K_{\text {softening }}-K_{\text {stiffening }}\right) v .
\end{aligned}
$$

We then substitute the effective stiffness expression given by Eqs. (A1) and (A2) in Eq. (A3) to obtain

$$
\begin{aligned}
{[1-\gamma} & \left.+\frac{3}{4} \Gamma\left(\Delta A_{\text {stiffening }}\right)^{2}\right] u^{\prime} \\
= & -\left[2+\frac{3}{4} \Gamma\left(\Delta A_{\text {stiffening }}-\Delta A_{\text {softening }}\right)^{2}-\Omega^{2}\right] v \\
& +\left[2 \gamma-\frac{3}{4} \Gamma\left(\Delta A_{\text {stiffening }}+\Delta A_{\text {softening }}\right)^{2}\right] u, \\
{\left[1-\gamma+\frac{3}{4} \Gamma\left(\Delta A_{\text {stiffening }}\right)^{2}\right] v^{\prime} } & \\
= & {\left[2+\frac{3}{4} \Gamma\left(\Delta A_{\text {stiffening }}-\Delta A_{\text {softening }}\right)^{2}-\Omega^{2}\right] u } \\
& -\left[2 \gamma-\frac{3}{4} \Gamma\left(\Delta A_{\text {stiffening }}+\Delta A_{\text {softening }}\right)^{2}\right] v .
\end{aligned}
$$

Using the continuum approximation, we can estimate the general strain amplitude in two consecutive springs as $\Delta A_{\text {softening }}=|u-v|$ and $\Delta A_{\text {stiffening }}=\left|u+v+u^{\prime}\right|$ or $\mid u+$ $v-v^{\prime} \mid$. We substitute these expressions in the equations above and neglect higher-order terms for small $\gamma, \Gamma, u^{\prime}$, and $v^{\prime}$, to get

$$
\begin{aligned}
& u^{\prime}=-\left(2-\Omega^{2}\right) v+2 \gamma u-\frac{3}{2} \Gamma\left(u^{3}+3 u v^{2}\right), \\
& v^{\prime}=\left(2-\Omega^{2}\right) u-2 \gamma v+\frac{3}{2} \Gamma\left(v^{3}+3 u^{2} v\right),
\end{aligned}
$$

which are exactly the same as Eq. (5) and proves that a linear dynamical system with effective stiffness could be used as a local mapping for the nonlinear dynamical system for all the frequencies inside the band gap.

\section{APPENDIX B: ANALYTICAL EXPRESSIONS FOR SOLITON AND EVANESCENT SOLUTIONS}

Soliton solutions. An integral of motion of the dynamical system given by Eq. (9) is

$$
E=2 \gamma u v\left[1-\frac{3 \Gamma}{4 \gamma}\left(u^{2}+v^{2}\right)\right]
$$

If we define an auxiliary function $g=u / v$, we can write the following equation:

$$
\left(\frac{d g}{d z}\right)^{2}=16 \gamma^{2} g^{2}-24 \Gamma g\left(1+g^{2}\right) E .
$$

We can then extract the integral of motion for the soliton solution from the phase portrait in Fig. 3(a). We substitute $(u, v)=(2 \sqrt{\gamma / 3 \Gamma}, 0)$ in Eq. (B1) since this point lies in the soliton trajectory. We obtain $E=0$, which we use in Eq. (B2) to get

$$
\frac{d g}{d z}= \pm 4 \gamma g .
$$

We now use the auxiliary function $g(z)=\exp ( \pm 4 \gamma z)$ and Eq. (B1) to obtain soliton profiles,

$$
\begin{aligned}
& u= \pm \sqrt{\frac{2 \gamma}{3 \Gamma}[1 \pm \tanh (4 \gamma z)]}, \\
& v= \pm \sqrt{\frac{2 \gamma}{3 \Gamma}[1 \mp \tanh (4 \gamma z)] .}
\end{aligned}
$$

Evanescent solutions. Modes shown in Figs. 3(d) and 3(f) lie on the $u=0$ and $v=0$ axes of the phase portrait. Therefore, to find the analytical form the solutions lying on the $v=0$ axis, we substitute $v=0$ in Eq. (9),

$$
\begin{aligned}
u^{\prime} & =2 \gamma u-\frac{3}{2} \Gamma u^{3}, \\
v^{\prime} & =0 .
\end{aligned}
$$

We define a new variable $y=1 / u^{2}$ and transform these equations to

$$
\begin{aligned}
& y^{\prime}=-4 \gamma y+3 \Gamma, \\
& v^{\prime}=0 .
\end{aligned}
$$

Clearly, these are two linear differential equations and can be solved easily. With the initial condition $(u, v)=\left(u_{0}, 0\right)$ at $z=$ $z_{0}$, we thus obtain the evanescent solution profile,

$$
\begin{aligned}
& u= \pm \frac{1}{\sqrt{y}}= \pm\left[\frac{3 \Gamma}{4 \gamma}+\left(\frac{1}{u_{0}^{2}}-\frac{3 \Gamma}{4 \gamma}\right) \exp \left[-4 \gamma\left(z-z_{0}\right)\right]\right]^{-1 / 2}, \\
& v=0 .
\end{aligned}
$$



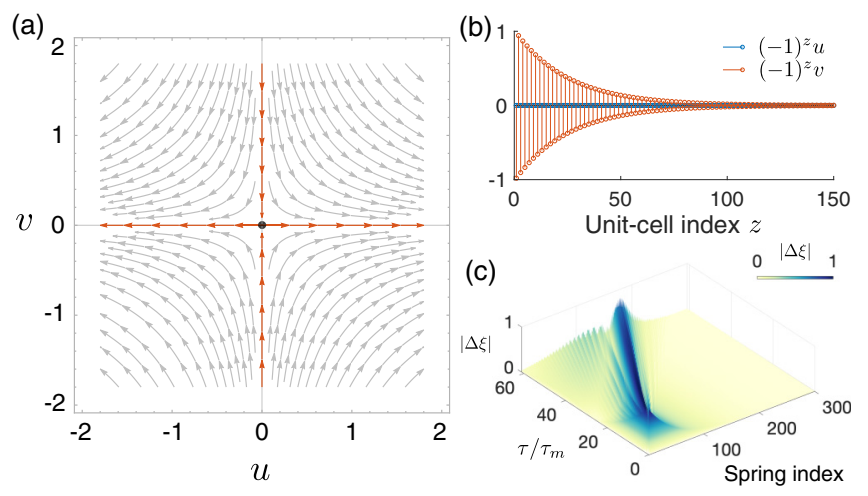

FIG. 8. (a) Phase portrait for a linear system $(\Gamma=0)$ showing steady-state solutions at the midgap frequency. There is a fixed point indicated by the black dot at the origin. Red arrows show the evanescent solutions in the bulk. (b) An evanescent mode solution decaying from the left corresponds to the solutions on $u=0$ axis. (c) Space-time evolution of the absolute value of strain when we use the aforementioned mode profile as an initial condition for a finite chain with the symmetry-preserving left boundary.

Note that these represent two solutions that correspond to a positive and negative sign of the initial condition $u_{0}$. Similarly, we can obtain solutions lying on $u=0$ axis with the initial condition $(u, v)=\left(0, v_{0}\right)$ at $z=z_{0}$,

$$
\begin{aligned}
& u=0 \\
& v= \pm\left[\frac{3 \Gamma}{4 \gamma}+\left(\frac{1}{v_{0}^{2}}-\frac{3 \Gamma}{4 \gamma}\right) \exp \left[4 \gamma\left(z-z_{0}\right)\right]\right]^{-1 / 2} .
\end{aligned}
$$

\section{APPENDIX C: PHASE PORTRAIT OF A LINEAR SYSTEM}

Here we discuss the features of the phase portrait obtained for a chain shown in Fig. 1(b), but with only linear springs. To this end, we substitute $\Gamma=0$ in Eq. (9) and obtain

$$
\begin{aligned}
& u^{\prime}=2 \gamma u, \\
& v^{\prime}=-2 \gamma v .
\end{aligned}
$$

These are two uncoupled first-order differential equations with a fixed point at $(u, v)=(0,0)$. We show the phase portrait in Fig. 8(a). By comparing it with the one for the nonlinear case in Fig. 3(a), we notice that its surrounding eight fixed points tend to go to infinity in the linear limit. As a result, we only see the evanescent modes in red from/to the fixed point at the origin. Thus, we would not expect any "plateau" in the mode shape since there is no fixed point at finite nonzero $u$ or $v$. We verify it by extracting a solution with the initial condition $\left(u_{0}, v_{0}\right)=(0,1)$ and plotting in Fig. 8(b). We observe that the evanescent solution asymptotically decays to zero displacements. Note that there are other evanescent solutions localized towards the right end, also with the nonlinear case in Fig. 3(a). We ignore them as our chain is assumed to be semi-infinite and we only focus on the left end as stated in the main text.

This bulk is topologically trivial as one can interpret from Figs. 2(b) and 3(a) in low amplitude (linear) limit. Therefore, the evanescent mode we obtained here is similar to the one in Fig. 3(d) topologically and this would not lead to a nonlinear edge mode for a finite chain with the symmetry-preserving boundary condition. We demonstrate this by full numerical simulation in Fig. 8(c), which shows significant scattering.

\section{APPENDIX D: OTHER FIXED POINTS IN THE PHASE PORTRAIT}

In Fig. 3(a), we observe four other fixed points in the region inside the soliton trajectories. These are $(u, v)=$ $( \pm \sqrt{\gamma / 3 \Gamma}, \pm \sqrt{\gamma / 3 \Gamma})$, which represent periodic solutions for the case when one spring (stiffening or softening) sees zero strain, and the other $\pm 2 \sqrt{\gamma / 3 \Gamma}$. As we have two types of nonlinear springs and their strain amplitudes can independently change, so it makes sense that this periodic solution would also lead to the same oscillation frequency $\left(\Omega_{m}\right)$, just as for the other fixed points on the $u=0$ and $v=0$ axes.

We also verify that these fixed points, and also their neighboring periodic orbits, would lead to solutions that obey $K_{\text {stiffening }}<K_{\text {softening }}$ for the entire length of the chain. Therefore, this represents a topologically trivial regime, surrounded by soliton trajectories, as also observed for the evanescent solutions in Sec. IV.

\section{APPENDIX E: EFFECTIVE STIFFNESS FOR SATURATING NONLINEARITY}

We take the force-deformation profile of stiffening springs,

$$
F(\Delta x)=(1+\gamma) k \Delta x-2 \gamma k \frac{\operatorname{erf}(v \Delta x)}{c v},
$$

and expand in power series, such that

$$
\begin{aligned}
F(\Delta x)= & (1-\gamma) k \Delta x \\
& +\frac{2 \gamma k}{v}\left[\frac{(v \Delta x)^{3}}{3}-\frac{(v \Delta x)^{5}}{5.2 !}+\frac{(v \Delta x)^{7}}{7.3 !}-\cdots\right] .
\end{aligned}
$$

Thus we write a general form

$$
F(\Delta x)=(1-\gamma) k \Delta x+k_{3}(\Delta x)^{3}-k_{5}(\Delta x)^{5}+\cdots,
$$

where the coefficient of $(\Delta x)^{n}$ is defined as

$$
k_{n}=2 \gamma k\left[\frac{v^{n-1}}{n\left(\frac{n-1}{2}\right) !}\right], \quad n=3,5,7, \ldots
$$

We also define a nondimensional parameter to indicate the extent of nonlinearity,

$$
\Gamma_{n}=a^{n-1} \frac{k_{n}}{k}=2 \gamma\left[\frac{(a v)^{n-1}}{n\left(\frac{n-1}{2}\right) !}\right], \quad n=3,5,7, \ldots .
$$

We now write the equations of motion in nondimensional form and employ a similar procedure as was done in Appendix A. Thus we obtain the lower band-edge frequency

$$
\begin{aligned}
\Omega_{l}^{2}= & 2(1-\gamma)+6 \Gamma_{3} A^{2}-20 \Gamma_{5} A^{4}+70 \Gamma_{7} A^{6} \\
& -252 \Gamma_{9} A^{8}+924 \Gamma_{11} A^{10}-\cdots \\
= & 2(1-\gamma)+2 \sum_{n=3,5, \ldots}(-1)^{(n+1) / 2} n C_{(n-1) / 2} \Gamma_{n} A^{n-1},
\end{aligned}
$$

where the Catalan number is defined as

$$
C_{m}=\frac{(2 m) !}{(m+1) ! m !} .
$$


Similarly, we can calculate the upper band-edge frequency

$$
\Omega_{u}^{2}=2(1+\gamma)-2 \sum_{n=3,5, . .}(-1)^{(n+1) / 2} n C_{(n-1) / 2} \Gamma_{n} A^{n-1} .
$$

We, therefore, obtain effective stiffnesses for the springs in terms of strain amplitude $\Delta A=2 A$,

$$
\begin{aligned}
K_{\text {stiffening }}(\Delta A) & =\frac{\Omega_{l}^{2}}{2} \\
& =(1-\gamma)+\sum_{n=3,5, \ldots}(-1)^{(n+1) / 2} n C_{(n-1) / 2} \Gamma_{n}\left(\frac{\Delta A}{2}\right)^{n-1}, \\
K_{\text {softening }}(\Delta A) & =\frac{\Omega_{u}^{2}}{2} \\
& =(1+\gamma)-\sum_{n=3,5, \ldots}(-1)^{(n+1) / 2} n C_{(n-1) / 2} \Gamma_{n}\left(\frac{\Delta A}{2}\right)^{n-1} .
\end{aligned}
$$

With this, we see how nonlinearity changes the effective stiffness of the springs. Increasing strain amplitude would make effective stiffnesses come closer to each other, and then go apart and saturate. The point (strain) where these stiffnesses would be equal, indicating the closure of the band gap, is determined by a graphical method in Fig. 5(a). This strain amplitude explains the plateau in the edge mode profile shown for this case.

[1] M. Z. Hasan and C. L. Kane, Colloquium: Topological insulators, Rev. Mod. Phys. 82, 3045 (2010).

[2] L. Lu, J. D. Joannopoulos, and M. Soljačić, Topological photonics, Nat. Photon. 8, 821 (2014).

[3] R. Süsstrunk and S. D. Huber, Classification of topological phonons in linear mechanical metamaterials, Proc. Natl. Acad. Sci. USA 113, E4767 (2016).

[4] G. Ma, M. Xiao, and C. T. Chan, Topological phases in acoustic and mechanical systems, Nat. Rev. Phys. 1, 281 (2019).

[5] G. Theocharis, N. Boechler, and C. Daraio, Nonlinear periodic phononic structures and granular crystals, in Acoustic Metamaterials and Phononic Crystals, edited by P. A. Deymier (Springer, Berlin, Heidelberg, 2013), pp. 217-251.

[6] M. I. Hussein, M. J. Leamy, and M. Ruzzene, Dynamics of phononic materials and structures: Historical origins, recent progress, and future outlook, Appl. Mech. Rev. 66, 040802 (2014).

[7] M. A. Porter, P. G. Kevrekidis, and C. Daraio, Granular crystals: Nonlinear dynamics meets materials engineering, Phys. Today 68(11), 44 (2015).

[8] B. Deng, P. Wang, Q. He, V. Tournat, and K. Bertoldi, Metamaterials with amplitude gaps for elastic solitons, Nat. Commun. 9, 3410 (2018).

[9] H. Yasuda, Y. Miyazawa, E. G. Charalampidis, C. Chong, P. G. Kevrekidis, and J. Yang, Origami-based impact mitigation via rarefaction solitary wave creation, Sci. Adv. 5, eaau 2835 (2019).

[10] F. Fraternali, G. Carpentieri, and A. Amendola, On the mechanical modeling of the extreme softening/stiffening response of axially loaded tensegrity prisms, J. Mech. Phys. Solids 74, 136 (2015).

[11] J. R. Raney, N. Nadkarni, C. Daraio, D. M. Kochmann, J. A. Lewis, and K. Bertoldi, Stable propagation of mechanical signals in soft media using stored elastic energy, Proc. Natl. Acad. Sci. USA 113, 9722 (2016).
[12] K. Bertoldi, V. Vitelli, J. Christensen, and M. van Hecke, Flexible mechanical metamaterials, Nat. Rev. Mater. 2, 17066 (2017).

[13] Y. Lumer, Y. Plotnik, M. C. Rechtsman, and M. Segev, SelfLocalized States in Photonic Topological Insulators, Phys. Rev. Lett. 111, 243905 (2013).

[14] M. J. Ablowitz, C. W. Curtis, and Y.-P. Ma, Linear and nonlinear traveling edge waves in optical honeycomb lattices, Phys. Rev. A 90, 023813 (2014).

[15] D. Leykam and Y. D. Chong, Edge Solitons in NonlinearPhotonic Topological Insulators, Phys. Rev. Lett. 117, 143901 (2016).

[16] Y. Lumer, M. C. Rechtsman, Y. Plotnik, and M. Segev, Instability of bosonic topological edge states in the presence of interactions, Phys. Rev. A 94, 021801(R) (2016).

[17] Y. Hadad, A. B. Khanikaev, and A. Alù, Self-induced topological transitions and edge states supported by nonlinear staggered potentials, Phys. Rev. B 93, 155112 (2016).

[18] D. D. Solnyshkov, O. Bleu, B. Teklu, and G. Malpuech, Chirality of Topological Gap Solitons in Bosonic Dimer Chains, Phys Rev. Lett. 118, 023901 (2017).

[19] D. A. Dobrykh, A. V. Yulin, A. P. Slobozhanyuk, A. N. Poddubny, and Y. S. Kivshar, Nonlinear Control of Electromagnetic Topological Edge States, Phys. Rev. Lett. 121, 163901 (2018).

[20] A. N. Poddubny and D. A. Smirnova, Ring Dirac solitons in nonlinear topological systems, Phys. Rev. A 98, 013827 (2018).

[21] R. S. Savelev, M. A. Gorlach, and A. N. Poddubny, Topological interface states mediated by spontaneous symmetry breaking, Phys. Rev. B 98, 045415 (2018).

[22] S. Kruk, A. Poddubny, D. Smirnova, L. Wang, A. Slobozhanyuk, A. Shorokhov, I. Kravchenko, B. LutherDavies, and Y. Kivshar, Nonlinear light generation in topological nanostructures, Nat. Nanotechnol. 14, 126 (2019). 
[23] R. K. Pal, J. Vila, M. Leamy, and M. Ruzzene, Amplitudedependent topological edge states in nonlinear phononic lattices, Phys. Rev. E 97, 032209 (2018).

[24] D. D. Snee and Y.-P. Ma, Edge solitons in a nonlinear mechanical topological insulator, Extreme Mech. Lett. 30, 100487 (2019).

[25] B. G.-g. Chen, N. Upadhyaya, and V. Vitelli, Nonlinear conduction via solitons in a topological mechanical insulator, Proc. Natl. Acad. Sci. USA 111, 13004 (2014).

[26] Y. Hadad, V. Vitelli, and A. Alu, Solitons and propagating domain walls in topological resonator arrays, ACS Photon. 4, 1974 (2017).

[27] W. P. Su, J. R. Schrieffer, and A. J. Heeger, Solitons in Polyacetylene, Phys. Rev. Lett. 42, 1698 (1979).

[28] C. L. Kane and T. C. Lubensky, Topological boundary modes in isostatic lattices, Nat. Phys. 10, 39 (2014).

[29] R. Chaunsali, E. Kim, A. Thakkar, P. G. Kevrekidis, and J. Yang, Demonstrating an In Situ Topological Band Transition in Cylindrical Granular Chains, Phys. Rev. Lett. 119, 024301 (2017).
[30] Y. Hadad, J. C. Soric, A. B. Khanikaev, and A. Alù, Selfinduced topological protection in nonlinear circuit arrays, Nat. Electron. 1, 178 (2018).

[31] J. Zak, Berry's Phase for Energy Bands in Solids, Phys. Rev. Lett. 62, 2747 (1989).

[32] P. Delplace, D. Ullmo, and G. Montambaux, Zak phase and the existence of edge states in graphene, Phys. Rev. B 84, 195452 (2011).

[33] B. Denardo, B. Galvin, A. Greenfield, A. Larraza, S. Putterman, and W. Wright, Observations of Localized Structures in Nonlinear Lattices: Domain Walls and Kinks, Phys. Rev. Lett. 68, 1730 (1992).

[34] Y. S. Kivshar, Class of localized structures in nonlinear lattices, Phys. Rev. B 46, 8652 (1992).

[35] G. Huang, J. Shen, and H. Quan, Noncutoff kinks in damped and parametrically driven nonlinear lattices, Phys. Rev. B 48, 16795 (1993).

[36] E. Prodan and H. Schulz-Baldes, Bulk and Boundary Invariants for Complex Topological Insulators, Mathematical Physics Studies (Springer International, Cham, 2016). 